\title{
Luminescence of free-standing versus matrix-embedded oxide-passivated silicon nanocrystals: The role of matrix-induced strain
}

Kateřina Kůsová, Lukáš Ondič, Eva Klimešová, Kateřina Herynková, Ivan Pelant, Stanislav Daniš, Jan Valenta, Mathieu Gallart, Marc Ziegler, Bernd Hönerlage, and Pierre Gilliot

Citation: Appl. Phys. Lett. 101, 143101 (2012); doi: 10.1063/1.4756696

View online: https://doi.org/10.1063/1.4756696

View Table of Contents: http://aip.scitation.org/toc/apl/101/14

Published by the American Institute of Physics

\section{Articles you may be interested in}

Silicon nanocrystals with ensemble quantum yields exceeding $60 \%$

Applied Physics Letters 88, 233116 (2006); 10.1063/1.2210788

Silicon quantum wire array fabrication by electrochemical and chemical dissolution of wafers Applied Physics Letters 57, 1046 (1990); 10.1063/1.103561

The structural and luminescence properties of porous silicon

Journal of Applied Physics 82, 909 (1997); 10.1063/1.366536

Charge transport in $\mathrm{Si}$ nanocrystal/ $\mathrm{SiO}_{2}$ superlattices

Journal of Applied Physics 113, 133703 (2013); 10.1063/1.4798395

Optical absorption and emission of silicon nanocrystals: From single to collective response Journal of Applied Physics 113, 143505 (2013); 10.1063/1.4799394

Influence of average size and interface passivation on the spectral emission of $\mathrm{Si}$ nanocrystals embedded in $\mathrm{SiO}_{2}$ Journal of Applied Physics 91, 798 (2002); 10.1063/1.1423768

\section{PHYSICS TODAY}

MANAGER'S GUIDE WHITEPAPERS
Accelerate R\&D with Multiphysics Simulation

\section{READ NOW}

PRESENTED BY

И $\subset$ OMSOL 


\title{
Luminescence of free-standing versus matrix-embedded oxide-passivated silicon nanocrystals: The role of matrix-induced strain
}

\author{
Kateřina Kůsová, ${ }^{1, a)}$ Lukáš Ondič,,${ }^{1,2,3}$ Eva Klimešová, ${ }^{1}$ Kateřina Herynková, ${ }^{1}$ Ivan Pelant, ${ }^{1}$ \\ Stanislav Daniš, ${ }^{3}$ Jan Valenta, ${ }^{3}$ Mathieu Gallart, ${ }^{2}$ Marc Ziegler, ${ }^{2}$ Bernd Hönerlage, ${ }^{2}$ \\ and Pierre Gilliot ${ }^{2}$ \\ ${ }^{1}$ Institute of Physics, Academy of Sciences of the Czech Republic, v.v.i., Cukrovarnická 10, 162 53, Prague 6, \\ Czech Republic \\ ${ }^{2}$ IPCMS-DON Unité Mixte, UMR 7504, CNRS-ULP, 23 rue du Loess, BP 43, 67034 Strasbourg Cedex 2, \\ France \\ ${ }^{3}$ Faculty of Mathematics and Physics, Charles University, Ke Karlovu 3, 12116 Prague 2, Czech Republic
}

(Received 8 June 2012; accepted 14 September 2012; published online 1 October 2012)

\begin{abstract}
We collect a large number of experimental data from various sources to demonstrate that free-standing (FS) oxide-passivated silicon nanocrystals (SiNCs) exhibit considerably blueshifted emission, by $200 \mathrm{meV}$ on average, compared to those prepared as matrix-embedded (ME) ones of the same size. This is suggested to arise from compressive strain, exerted on the nanocrystals by their matrix, which plays an important role in the light-emission process; this strain has been neglected up to now as opposed to the impact of quantum confinement or surface passivation. Our conclusion is also supported by the comparison of low-temperature behavior of photoluminescence of matrix-embedded and free-standing silicon nanocrystals. (c) 2012 American Institute of Physics. [http://dx.doi.org/10.1063/1.4756696]
\end{abstract}

Silicon nanocrystals (SiNCs) are a material of intense scientific interest due to their prospective applications as nanoscale light emitters both in optoelectronics ${ }^{1}$ and as fluorescent markers ${ }^{2}$ in bio-imaging, and, moreover, for enhancing the efficiency of photovoltaic solar cells. ${ }^{3}$ SiNCs emit light quite efficiently in contrast to their bulk counterpart. The origin of this light emission is still not fully understood in detail. It is generally believed that their slow red/orange emission (the so-called $\mathrm{S}$ band) results from indirect $\Delta_{1}-\Gamma_{25^{\prime}}$ transition, ${ }^{4,5}$ being influenced by a complex interplay of quantum confinement ${ }^{6}$ and surface terminating species. ${ }^{7}$ The effect of quantum confinement is most easily demonstrable in SiNCs with "intrinsic" hydrogen surface termination as their photoluminescence (PL) is easily tunable between green and deep red emission (550$800 \mathrm{~nm}$ ) by changing the size of the crystalline core. ${ }^{8}$

Since hydrogen surface termination is highly unstable in air and such nanocrystals are strongly prone to oxidation,, 910 such nanocrystals have to be surface-treated. The surface capping layer of stable SiNC can be either made up by silicon oxide or by a specially prepared layer of organic molecules if oxidation is intentionally avoided. ${ }^{9}$ The detailed chemical composition of the surface terminating layer significantly influences the spectral PL position of the sample; therefore, here, we focus on oxide-capped SiNCs to minimize the effect of surface chemistry on the proposed reasoning.

In oxide-passivated SiNCs, the oxide-related surface states profoundly influence $\mathrm{PL}^{7,10}$ because of the formation of discrete states inside the bandgap. For example, the emission of green-emitting (2.35 eV) hydrogen-terminated SiNCs shifts to the red spectral region $(1.85 \mathrm{eV})$ upon oxidation. ${ }^{10}$ PL of oxide-capped SiNCs is also size-tunable to some extent: e.g., spectral shifts from 1.32 to $2.4 \mathrm{eV}$ were observed upon the decrease in size from 7.8 to $2 \mathrm{~nm}$ by Ledoux

${ }^{\text {a)} E l e c t r o n i c ~ m a i l: ~ k u s o v a @ f z u . c z . ~}$ et al., ${ }^{11}$ or spectral shifts from 1.2 to $1.36 \mathrm{eV}$ upon size decrease from 9 to $4.7 \mathrm{~nm}$ were observed by Takeoka et al. ${ }^{12}$ However, the wavelength tunability is somewhat limited when compared to hydrogen termination ${ }^{13}$ and, even if only oxide-capped SiNCs are considered, their PL is influenced by defects and/or the core/shell interface. ${ }^{14,15}$

In general, SiNCs can be prepared via a wide range of techniques. For the purposes of this article, it is useful to divide SiNC samples into those prepared as free-standing (FS) or matrix-embedded (ME). A wide variety of colloidal FS SiNCs can be prepared by chemical synthesis, ${ }^{9}$ but these nanocrystals are quite exclusively very small $(<1.8 \mathrm{~nm})$ with organic surface capping, and therefore their PL emission does not include the $\mathrm{S}$ band, being situated in the UV/blue spectral region. Focusing on FS oxide-passivated SiNCs, they can be prepared by various kinds of wet etching (either electrochemical HF-based etching ${ }^{7,16}$ or simple chemical $\mathrm{HF} / \mathrm{HNO}_{3}$ etching $^{17}$ of variously prepared $\mathrm{Si}$ powders constituted by particles with sizes $<30 \mathrm{~nm}$, further reducing the core sizes by chemical etching) or synthesis in plasma from silane. ${ }^{11,18}$ The etching or synthesis is then followed by slow oxidation in ambient conditions; the FS powder can also be deposited on a substrate or form a suspension/colloidal dispersion in liquid. ME oxide-capped SiNCs, on the other hand, can be prepared by the deposition of $\mathrm{SiO}_{x} / \mathrm{SiO}_{2}$ superlattices ${ }^{3,19,20}$ or $\mathrm{Si}$-rich $\mathrm{SiO}_{2},{ }^{12,14,21,22}$ by the implantation of $\mathrm{Si}$ ions into $\mathrm{SiO}_{2}$ matrix, ${ }^{23}$ or by chemical synthesis in a polymer-based matrix. ${ }^{24}$ After the deposition or implantation step, the samples are typically annealed $\left(>1000^{\circ} \mathrm{C}\right)$, which results in the formation of $\mathrm{SiNC}$, either as small inclusions inside an $\mathrm{SiO}_{2}$-based matrix or as densely packed thin films overgrown with $\mathrm{SiO}_{2}$.

All the references mentioned in the previous paragraph report reliable data on the room-temperature PL spectra of oxide-passivated SiNCs along with the corresponding particle sizes. All these data, measured on more than 100 samples 


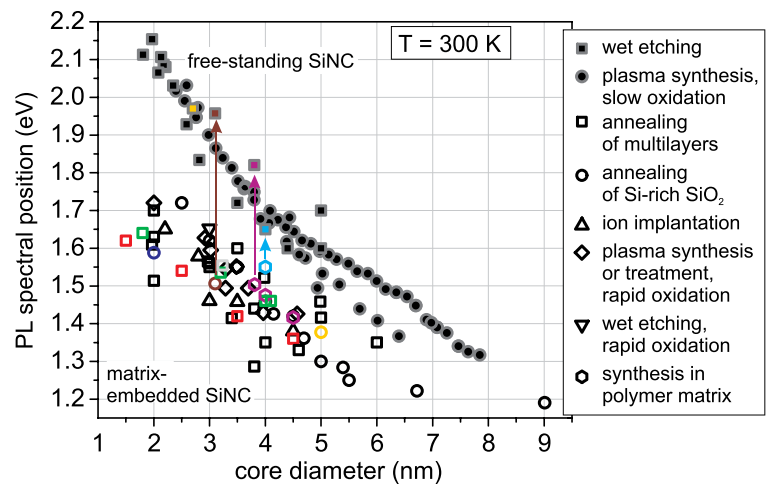

FIG. 1. Summary of experiments reporting photoluminescence spectral maximum as a function of size of oxidized SiNCs $(T=300 \mathrm{~K})$. Grayoutlined solid symbols correspond to samples prepared as FS (wet etching taken from Refs. 7, 16, and 17; plasma synthesis and slow oxidation taken from Refs. 11 and 18), open symbols denote samples prepared as ME $\left(\mathrm{SiO}_{x} / \mathrm{SiO}_{2}\right.$ superlattices taken from Refs. 3, 19, and 20, Si-rich $\mathrm{SiO}_{2}$ taken from Refs. 12, 14, 21, and 22; Si ion implantation taken from Ref. 23; plasma synthesis taken from Ref. 25; electrochemical etching followed by thermal oxidation taken from Ref. 26; and chemical synthesis in polymerbased matrix taken from Ref. 24). Samples discussed in more detail are drawn in color, symbols connected by arrows were prepared as ME, but liberated from the matrix and their PL as FS was also measured.

by various groups worldwide, can be compiled to Fig. 1. In this plot, one can easily see the distinction between ME, denoted by open symbols, and FS samples, denoted by solid symbols: even if both the size and the type of surface passivation are the same, the PL of ME samples is considerably redshifted in comparison with the FS samples, roughly by $200 \mathrm{meV}$. This significant difference is easily visible with the naked eye, just comparing the color of PL emitted by a ME and FS sample as shown in Figs. 2(a) and 2(b).

A factor playing an important role in this spectral shift is the influence of compressive strain exerted on the ME SiNCs by their matrix. When growing inside a matrix, a ME SiNC cannot grow freely, but is constantly compressed by the matrix. On the other hand, spontaneous growth of oxide on the surface of a FS nanocrystal under ambient conditions does not exert any strain on the nanocrystalline core. However, "forced" oxidation, e.g., under high temperature, may cause the growth of a compressive outer oxide shell or even a thin-film oxide layer burying the originally FS SiNCs, causing such nanocrystals to behave as ME ones. This is the case, e.g., for samples prepared by plasma synthesis from silane by Takagi et al., ${ }^{25}$ by reactive ion etching in plasma by
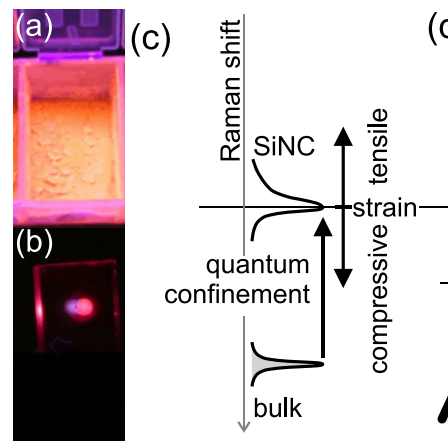

(d)

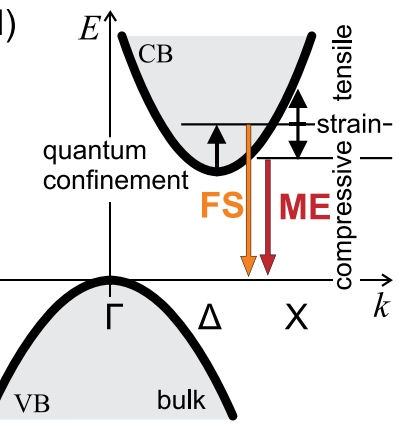

FIG. 2. Photos of PL of a (a) free-standing and (b) matrix-embedded SiNC samples. Schematics of the influence of quantum confinement and strain on (c) Raman spectra of optical phonon of Si (nano)crystal and (d) bandstructure of silicon.
Ray et al., ${ }^{17}$ and by electrochemical etching by Kanemitsu et $a .^{26}$ included in Fig. 1 as ME ones. On the other hand, it was shown that compressive stress could be relaxed, e.g., upon local laser annealing of ME SiNCs prepared by rapid thermal annealing of $\mathrm{Si} / \mathrm{SiO}_{2}$ superlattices. ${ }^{27}$ Therefore, it is important to take into account all the steps of the preparation process to correctly determine if the "matrix-induced" compression is present in the investigated sample and if it possibly influences its PL. Inevitably, different levels of stress will be present in different samples; this is indirectly supported also by the larger variability in the PL spectral position versus core diameter dependence in the ME-prepared SiNCs when compared to the FS-prepared ones in Fig. 1.

In some cases, namely in samples prepared by annealing of $\mathrm{Si}$-rich $\mathrm{SiO}_{2}$ layers by Matsuhisa et al. ${ }^{21}$ (brown symbols in Fig. 1) and by synthesis in a polymer-based matrix reported on in Ref. 24 (cyan and magenta symbols in Fig. 1, respectively), the ME SiNCs were liberated from the $\mathrm{SiO}_{2}$ matrix by HF etching, giving rise to FS SiNC samples (the correspondingly colored solid points in Fig. 1 are connected by arrows). Evidently, this ME $\rightarrow$ FS transition is accompanied by a PL blueshift: Fig. 1 demonstrates that the PL spectral position of ME SiNCs after etching well accords with that of the FS-prepared SiNCs. ${ }^{28}$ This blueshift, whose origin is, interestingly enough, not commented on in the original studies, confirms the connection between the PL shift and the matrix: when a nanocrystal is released from the matrix, the matrix-induced compressive strain is relaxed and is no longer present, giving rise to a FS SiNC.

The idea that compressive stress is present in ME SiNCs is not completely new, but it comes to mind much more naturally in connection with Raman measurements than with PL. The combined influence of quantum confinement and strain on the SiNC phonon Raman peak is quite complex (see Fig. 2(c)) and, therefore, extreme care has to be taken during such an analysis. Due to the possibility of systematic error, samples in which the size was determined solely by analysis of SiNC Raman spectra were not included in Fig. 1.

Some analyses of Raman measurements of ME oxidepassivated SiNCs detected the presence of compressive stress. Namely, Arguirov et al. ${ }^{27}$ observed an unusual up-shift in the ME SiNC phonon Raman frequency of SiNCs prepared by annealing of $\mathrm{Si} / \mathrm{SiO}_{2}$ superlattices, implying that in this case, the stress is compressive regardless of the used confinement model. The corresponding deduced compressive stress amounted to $\sim 5 \mathrm{GPa}$. Indeed, PL spectra of samples prepared by the same group and reported on by Rölver et al. ${ }^{29}$ exhibit a significantly redshifted PL maximum (please note that the same type of samples is later discussed in Fig. 5(b)). Furthermore, Hernándeź et al. ${ }^{30}$ identified compressive stresses of 3.5 GPa in ME SiNCs prepared by annealing of substechiometric $\mathrm{SiO}_{x}$ films; the maximum of the PL spectrum of the corresponding sample ${ }^{22}$ is drawn as open yellow circle in Fig. 1. Zatryb et al. ${ }^{31}$ confirmed somewhat lower levels of compressive stress between 0.4 and $0.8 \mathrm{GPa}$ in ME SiNCs prepared by annealing of $\mathrm{Si} / \mathrm{SiO}_{2}$ superlattices, PL of the corresponding sample ${ }^{19}$ is denoted by open light gray square in Fig. 1. Surprisingly, lattice contraction corresponding to stress of several GPa was confirmed also by HRTEM measurements of SiNCs prepared by inert gas arc evaporation, ${ }^{32}$ and the 
presence of stress was confirmed by $\mathrm{x}$-ray measurements in ME SiNC samples based on superlattices. ${ }^{33}$

Stress can be found also in cases slightly different from but analogical to oxide-passivated ME SiNCs. For example, SiNCs prepared by ion implantation to a sapphire matrix ${ }^{34}$ and subsequent annealing exhibited a small lattice contraction of $0.04 \%-0.11 \%$, corresponding to compressive stresses around $2 \mathrm{GPa}$. Moreover, the compressive effect of the matrix was also identified in $\mathrm{CdS}_{x} \mathrm{Se}_{1-x}$ nanocrystals ${ }^{35}$ via Raman measurements, and the comparison of Raman spectra of oxide-passivated $\mathrm{ME} \mathrm{SiNCs}^{36}$ with alkylated FS SiNCs also suggest compression by the matrix (although in this case, SiNCs with different surface passivations are studied). On the other hand, porous-silicon-based SiNC layers were found to exhibit lattice expansion ${ }^{37}$ by HRTEM measurements.

Although the presence of stress in SiNCs samples was considered in the past, rarely has it been connected with the PL properties; such a connection was only indicated by theoretical calculations of very small clusters ${ }^{38}$ consisting of less than $100 \mathrm{Si}$ atoms. Another theoretical calculation studying stress in SiNCs, which, however, disregards the implications for PL, was carried out by Yilmaz et al. ${ }^{39}$

The concept that matrix-induced compressive stress can be in many cases one of important factors determining the spectral position of SiNC samples can be inferred from basic solid state physics. If we consider that the PL in oxidized SiNCs arises from indirect $\Delta_{1}-\Gamma_{25^{\prime}}$ transitions in the silicon bandstructure, ${ }^{4,5}$ we can calculate the compressive strain necessary for down-shifting the $\Delta_{1}$ minimum by $200 \mathrm{meV}$, the value determined from Fig. 1. Compared to bulk Si, bandgap energy in SiNCs is increased as the $\Delta_{1}$ conductionband minimum up-shifts with decreasing size of the nanocrystal due to quantum confinement. ${ }^{40}$ Strain can then either further up-shift or down-shift this energy gap depending on its sign, see Fig. 2(d); the effect of strain on the quantumconfinement energy, though, will be negligible because strain-induced volume changes will be very small. The effect of strain on the bandstructure of solids is then usually expressed in terms of deformation potential ${ }^{41} a_{n}(\mathbf{k})$

$$
\delta E_{n}(\mathbf{k})=a_{n}(\mathbf{k})(\delta V / V)
$$

which links the shift of the energy of a particular band extreme $\delta E_{n}(\mathbf{k})$ with the relative change in volume $\delta V / V$. To gain a simple estimate of the order of magnitude of this effect, we can use the deformation potential values for bulk $\mathrm{Si}$, being ${ }^{41}$ $a(\Delta)=14 \mathrm{eV}$ for the indirect bandgap. This implies that the energy shift of $200 \mathrm{meV}$ corresponds to the volume change of the primitive cell by as little as $1.4 \%$. This value translates into a mere $0.7 \%$ change in lattice constant $(\sqrt[3]{0.986}=0.993)$. Still using the bulk $\mathrm{Si}$ approximation, stress $\delta p$ of the order of $\sim 2 \mathrm{GPa}$ is needed to induce such a change in volume (bulk modulus of bulk Si $K=\delta p /(\delta V / V) \sim 100 \mathrm{GPa}$ is a good approximation of that of nanocrystals ${ }^{5,42}$ ). This is a reasonable number, because it is well below the first pressure-induced phase transition in silicon ${ }^{43}$ from diamond to $\beta$-tin structure, which occurs at $12 \mathrm{GPa}$ and which would certainly be easily detectable in HRTEM imaging. Our estimate of $\sim 2 \mathrm{GPa}$ also well accords with the stress values measured by various groups as mentioned above.
In order to further support the proposed idea of matrixinduced strain, we carried out three types of experiments to complement the above compilation of literature data. First, apart from $\mathrm{SiNC}$ samples prepared from $\mathrm{Si} / \mathrm{SiO}_{2}$ superlattices and substechiometric $\mathrm{SiO}_{x}$ films, compressive stress can be found also in ion-implanted samples, as supported by our X-ray diffraction ${ }^{44}$ (XRD) measurements presented in Fig. 3. Lattice constant of $\mathrm{Si}$ in SiNCs was determined from the position of the $2 \theta$ angle of observed diffraction maximum corresponding to the (220) diffraction line. The maximum of the diffraction peak was measured at $2 \theta=(47.556 \pm 0.002)^{\circ}$, which gives the lattice parameter $(5.40 \pm 0.01) \AA$ (the lattice parameter of bulk silicon $a_{\mathrm{Si}}=5.431 \AA$ would correspond to the diffraction peak observed at a diffraction angle of $2 \theta=47.3^{\circ}$ ). Thus, the lattice parameter is contracted by $\sim 0.6 \%$, which very well accords with the above estimate of lattice contraction based on deformation potential. Correspondingly, the PL spectrum of the same sample (inset in Fig. 3) peaks at $860 \mathrm{~nm}$, which is a value considerably redshifted compared to FS samples.

Second, we addressed the issue if a nanocrystalline material behaves analogically to bulk when it comes to compression. Our measurements of the dependence of PL spectra of ethanoic suspensions of FS SiNCs on external hydrostatic pressure in a diamond cell ${ }^{44}$ are presented in Fig. 4. The PL maximum indeed redshifts with compressive pressure, this shift is, however, somewhat lower when compared to our above estimate. Very similar shifts of PL maxima of alkanecapped FS SiNCs have been reported recently. ${ }^{5}$ More effects can be responsible for this difference. First, theoretical calculations of strain fields in ME SiNCs suggest ${ }^{39}$ that stress is inhomogeneously distributed in the volume of an oxidecapped SiNC, being more compressive near the nanocrystal's surface. In oxide-capped SiNCs, the optical excitation is trapped at the surface, therefore, it might locally "feel" higher compressive stresses than those measured by Raman, HRTEM, or XRD, being averaged over the whole core. Second, the microscopic composition of the surface terminating layer can be to a certain extent different in the ME and FS

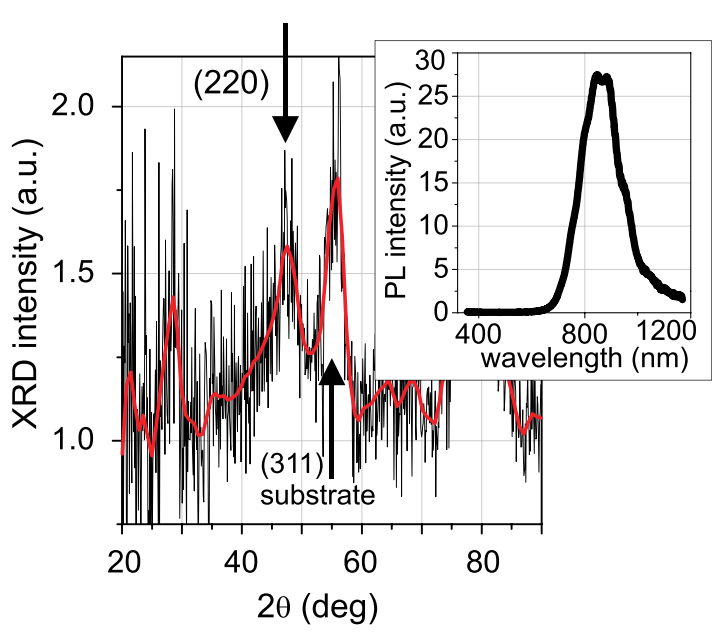

FIG. 3. XRD measurements of ion-implanted ME SiNCs. Raw data (black curve) were smoothed by cubic spline (red curve). The (220) Bragg peak originates in SiNCs, the (311) Bragg peak comes from the substrate, the remaining peaks are false peaks arising from the subtraction of background. The inset shows PL spectrum of the same sample. 


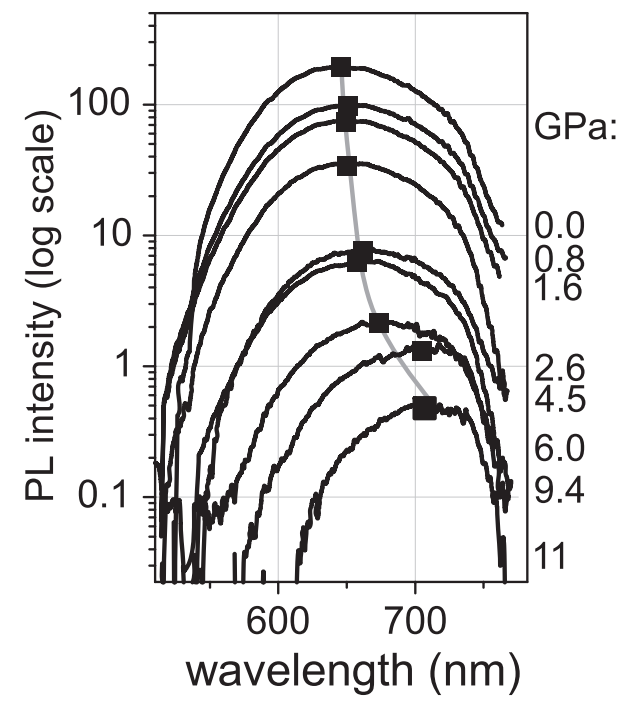

FIG. 4. Changes in PL spectra of FS SiNCs with external applied pressure, the squares correspond to the maximum of a Gaussian fit.

samples, also giving rise to a redshift; this scenario is supported by the fact that SiNC samples annealed at different ambients exhibit spectral shifts. ${ }^{14}$

Last, the difference between ME- and FS-prepared SiNCs can be further tested using the measurements of temperature dependence of PL. Several experiments ${ }^{14,20,45,46}$ have already reported on the temperature dependence of PL for ME samples. All of them concluded that the PL blueshift with decreasing temperature behaves very similarly to that of bulk Si, i.e., that PL blueshifts by about $50 \mathrm{meV}$ when the temperature drops from 300 to $3 \mathrm{~K}$, see Fig. 5(b). (The same samples as those in Fig. 5(b) are also included in roomtemperature PL in Fig. 1; colors of the symbols in the two figures are chosen to match.)

Our measurements of the temperature dependence of PL of FS SiNC samples ${ }^{44}$ suggest that significantly larger blueshift occurs at FS-prepared SiNC. Fig. 5(a) presents selected PL spectra (smoothed, corrected for the spectral sensitivity of the setup) of FS SiNCs measured at different temperatures, while Fig. 5(b) plots the temperature dependence of energy shift of PL maximum with regards to the lowest experimentally attainable temperatures for our measurements of FS samples along with the literature data on ME samples. ${ }^{14,20}$ Clearly, the PL blueshift is significantly larger for FS SiNCs, of about $180 \mathrm{meV}$ with respect to the ME value of $50 \mathrm{meV}$.

This difference can once again be explained by the presence of strain, which emerges in the FS sample when it is cooled down. It is obvious that a FS SiNC with a silica outer shell will tend to shrink with decreasing temperature. ${ }^{47}$ Judging simply from the thermal coefficients of expansion for bulk silicon and silica ( 3 vs. $0.3 \times 10^{-6} \mathrm{~K}^{-1}$ ), we can see that the silicon core will have much stronger tendency to shrink than the outer shell. Therefore, the volume change of the "hollow" $\mathrm{SiO}_{2}$ shell can be neglected. Unlike the bulk modulus, ${ }^{42}$ however, coefficient of thermal expansion $\alpha$ in a nanocrystalline material can be expected to be several times higher than that in bulk ${ }^{48}$ (i.e., $\alpha_{\mathrm{SiNC}} \approx 3 \times 3 \times 10^{-6} \mathrm{~K}^{-1}=9 \times 10^{-6} \mathrm{~K}^{-1}$ ) due to non-negligible influence of the surface. Consequently, the volume change of a nanocrystal when the temperature changes

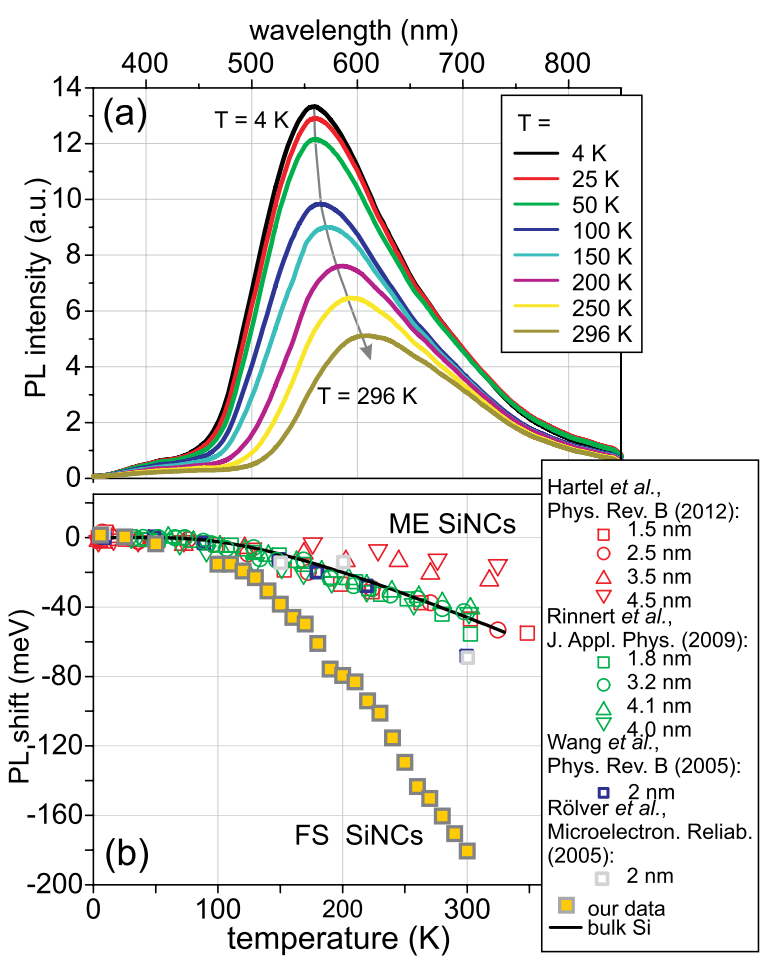

FIG. 5. (a) Measured spectra of FS SiNCs, the arrow denotes increasing temperature. (b) Temperature dependence of the PL shift with respect to the spectral position at the lowest experimental temperatures. Data of ME SiNCs (open symbols) are taken from the literature (see the legend), data of FS SiNCs (solid symbols) correspond to spectral positions from panel (a). Bulk Si temperature dependence is drawn in black line for comparison. Colors correspond to those in Fig. 1.

by $300 \mathrm{~K}$ can be estimated to be $\left(1-9 \times 10^{-6} \mathrm{~K}^{-1} \times 300 \mathrm{~K}\right)^{3}$ $=0.992$, i.e., the core has a tendency to shrink by about $0.8 \%$, but this shrinkage is prevented by the outer shell. If we take into account the above "calibration" based on deformation potential, we can estimate that this effect should induce tensile strain acting on the core of the nanocrystal corresponding to the shift in PL energy of about $110 \mathrm{meV}$.

On the other hand, ME SiNCs are under compressive strain of the matrix. This compressive strain relaxes on cooling, however, it is never completely lifted (compare the volume change of $1.8 \%$ due to matrix compression versus the much smaller $0.8 \%$ change due to temperature-induced tensile strain in FS SiNCs). Therefore, the PL energy shift in ME SiNCs with the decrease in temperature from 300 to $5 \mathrm{~K}$ will be driven by the inherent properties of the silicon lattice, i.e., will be roughly the same as in bulk $\mathrm{Si}(\sim 50 \mathrm{meV})$, whereas in the FS SiNCs, the tensile strain adds up to this value and the overall PL energy shift will amount to $\sim 160 \mathrm{meV}$, which is in reasonable accordance with our experimental results (see Fig. 5).

In conclusion, we show that a clear distinction exists between oxide-passivated SiNCs prepared as free-standing and matrix embedded. Data from the literature summarizing the size dependence of PL spectral position show that the ME SiNC samples are systematically redshifted by about $200 \mathrm{meV}$ when compared to FS samples of roughly the same size. We propose that non-negligible compressive stress ( $\sim 2 \mathrm{GPa}$ ) exerted on the SiNC by the matrix is an important factor inducing this redshift (in addition to the traditionally considered effect of surface capping layer). To support the 
proposed idea, we complement literature data by three types of dedicated experiments, studying XRD of ME SiNCs, pressure-dependence of PL of FS SiNCs, and carrying out temperature-dependent PL measurements on FS SiNCs. Importantly, the role of matrix-induced strain should not be overlooked in analyses of SiNC phonon mode in Raman measurements.

Financial support was provided by GAAVČR (Grant Nos. KJB100100903 and IAA101120804), GAUK (Grant No. 73910) and GAČR (Grant No. P204/12/P235), and the EC FP7 program (project NASCEnT, No. 245977). This work was also supported by the scholarship "thèse en cotutelle" of the Ministry of Education of France. We thank professor R. Elliman from Australian National University in Canberra for providing the ion-implanted sample.

${ }^{1}$ N. Daldosso and L. Pavesi, Laser Photon. Rev. 3, 508 (2009).

${ }^{2}$ J.-H. Park, L. Gu, G. von Maltzahn, E. Ruoslahti, S. N. Bhatia, and M. J. Sailor, Nature Mater. 8, 331 (2009).

${ }^{3}$ G. Conibeer, M. Green, R. Corkish, Y. Cho, E.-C. Cho, C.-W. Jiang, T. Fangsuwannarak, E. Pink, Y. Huang, T. Puzzer, T. Trupke, B. Richards, A. Shalav, and K.-L. Lin, Thin Solid Films 511-512, 654 (2006).

${ }^{4}$ D. Kovalev, H. Heckler, M. Ben-Chorin, G. Polisski, M. Schwartzkopff, and F. Koch, Phys. Rev. Lett. 81, 2803 (1998).

${ }^{5}$ D. C. Hannah, J. Yang, P. Podsiadlo, M. K. Chan, A. Demortiére, D. J. Gosztola, V. B. Prakapenka, G. C. Schatz, U. Kortshagen, and R. D. Schaller, Nano Lett. 12, 4200 (2012).

${ }^{6}$ L. Canham, Appl. Phys. Lett. 57, 1046 (1990).

${ }^{7}$ M. V. Wolkin, J. Jorne, P. M. Fauchet, G. Allan, and C. Delerue, Phys. Rev. Lett. 82, 197 (1999).

${ }^{8}$ A. Gupta, M. T. Swihart, and H. Wiggers, Adv. Funct. Mater. 19, 696 (2009).

${ }^{9}$ N. Shirahata, Phys. Chem. Chem. Phys. 13, 7284 (2011).

${ }^{10}$ K. Dohnalová, K. Kůsová, and I. Pelant, Appl. Phys. Lett. 94, 211903 (2009).

${ }^{11}$ G. Ledoux, J. Gong, F. Huisken, O. Guillois, and C. Reynaud, Appl. Phys. Lett. 80, 4834 (2002).

${ }^{12}$ S. Takeoka, M. Fujii, and S. Hayashi, Phys. Rev. B 62, 16820 (2000).

${ }^{13}$ K. Dohnalová, L. Ondič, K. Kůsová, I. Pelant, J. L. Rehspringer, and R.-R. Mafouana, J. Appl. Phys. 107, 053102 (2010).

${ }^{14}$ X. X. Wang, J. G. Zhang, L. Ding, B. W. Cheng, W. K. Ge, J. Z. Yu, and Q. M. Wang, Phys. Rev. B 72, 195313 (2005).

${ }^{15}$ J. Heitmann, F. Müller, M. Zacharias, and U. Gösele, Adv. Mater. 17, 795 (2005).

${ }^{16}$ K. Dohnalová, I. Pelant, K. Kůsová, P. Gilliot, M. Gallart, O. Crégut, J.-L. Rehspringer, B. Hönerlage, T. Ostatnický, and S. Bakardjieva, New J. Phys. 10, 063014 (2008).

${ }^{17}$ A. Vladimirov, S. Korovin, A. Surkov, E. Kelm, and V. Pustovoy, Laser Phys. 21, 830 (2011); A. Gupta and H. Wiggers, Nanotechnology 22, 055707 (2011); M. Ray, S. M. Hossain, R. F. Klie, K. Banerjee, and S. Ghosh, Nanotechnology 21, 505602 (2010).

${ }^{18}$ M. Ehbrecht, B. Kohn, F. Huisken, M. A. Laguna, and V. Paillard, Phys. Rev. B 56, 6958 (1997).

${ }^{19}$ M. Zacharias, J. Heitmann, R. Scholz, U. Kahler, M. Schmidt, and J. Bläsing, Appl. Phys. Lett. 80, 661 (2002); S. Kim, D. H. Shin, D. Y. Shin, C. O. Kim, J. H. Park, S. B. Yang, S.-H. Choi, S. J. Yoo, and J.-G. Kim, J. Nanomater. 2012, 572746 (2012); L. Khriachtchev, S. Novikov, J. Lahtinen, and M. Räsänen, J. Phys.: Condens. Matter. 16, 3219 (2004); L. X. Yi, J. Heitmann, R. Scholz, and M. Zacharias, Appl. Phys. Lett. 81, 4248 (2002); X. Yu, R. Zhang, Z. Xu, D. Zhang, H. Zhao, Y. Zheng, and L. Chen, J. Non-Cryst. Solids 357, 3524 (2011); M. Cazzanelli, D. NavarroUrriós, F. Riboli, N. Daldosso, L. Pavesi, J. Heitmann, L. X. Yi, R. Scholz, M. Zacharias, and U. Gösele, J. Appl. Phys. 96, 3164 (2004); X. J. Hao, A. P. Podhorodecki, Y. S. Shen, G. Zatryb, J. Misiewicz, and M. A. Green, Nanotechnology 20, 485703 (2009).

${ }^{20}$ H. Rinnert, O. Jambois, and M. Vergnat, J. Appl. Phys. 106, 023501 (2009); A. M. Hartel, S. Gutsch, D. Hiller, and M. Zacharias, Phys. Rev. B 85, 165306 (2012).

${ }^{21}$ K. Matsuhisa, M. Fujii, K. Imakita, and S. Hayashi, J. Lumin. 132, 1157 (2012); in this case, the size of the nanocrystal for Fig.1 is taken from [Y.
Kanzawa, T. Kageyama, S. Takeoka, M. Fujii, S. Hayashi, and K. Yamamoto, Solid State Commun. 102, 533 (1997)].

${ }^{22}$ W. D. A. M. de Boer, D. Timmerman, K. Dohnalova, I. N. Yassievich, H. Zhang, W. J. Buma, and T. Gregorkiewicz, Nat. Nanotechnol. 5, 878 (2010); Y. Lebour, P. Pellegrino, S. Hernández, A. Martínez, E. Jordana, J.-M. Fedeli, and B. Garrido, Physica E 41, 990 (2009).

${ }^{23}$ S. Guha, J. Appl. Phys. 84, 5210 (1998); L. Pavesi, L. D. Negro, L. Mazzoleni, G. Franzò, and F. Priolo, Nature 408, 440 (2000); G. A. Kachurin, S. G. Cherkova, D. V. Marin, R. A. Yankov, and M. Deutschmann, Nanotechnology 19, 355305 (2008); O. Korotchenkov, A. Podolian, V. Kuryliuk, B. Romanyuk, V. Melnik, and I. Khatsevich, J. Appl. Phys. 111, 063501 (2012).

${ }^{24}$ E. J. Henderson, J. A. Kelly, and J. G. C. Veinot, Chem. Mater. 21, 5426 (2009); C. M. Hessel, E. J. Henderson, and J. G. C. Veinot, Chem. Mater. 18, 6139 (2006).

${ }^{25}$ H. Takagi, H. Ogawa, Y. Yamazaki, A. lshizaki, and T. Nakagiri, Appl. Phys. Lett. 56, 2379 (2000).

${ }^{26}$ Y. Kanemitsu, T. Futagi, T. Matsumoto, and H. Mimura, Phys. Rev. B 49, 14732 (1994).

${ }^{27}$ T. Arguirov, T. Mchedlidze, M. Kittler, R. Rölver, B. Berghoff, M. Först, and B. Spangenberg, Appl. Phys. Lett. 89, 053111 (2006).

${ }^{28}$ The etching procedure can be connected with some size shrinkage and a subsequent PL blueshift. However, this size shrinkage should definitely not be larger than $\sim 0.3 \mathrm{~nm}$ if the nanoparticles are not intentionally left in the HF acid for too long.

${ }^{29}$ R. Rölver, S. Bruninghoff, M. Forst, B. Spangenberg, and H. Kurz, J. Vac. Sci. Technol. B 23, 3214 (2005).

${ }^{30}$ S. Hernández, A. Martínez, P. Pellegrino, Y. Lebour, B. Garrido, E. Jordana, and J. M. Fedeli, J. Appl. Phys. 104, 044304 (2008).

${ }^{31}$ G. Zatryb, A. Podhorodecki, X. J. Hao, J. Misiewicz, Y. S. Shen, and M. A. Green, Nanotechnology 22, 335703 (2011).

${ }^{32}$ H. Hofmeister and P. Ködderitzsch, Nanostruct. Mater. 12, 203 (1999).

${ }^{33}$ M. Zacharias, J. Blasing, P. Veit, L. Tsybeskov, K. Hirschman, and P. M. Fauchet, Appl. Phys. Lett. 74, 2614 (1999).

${ }^{34}$ S. Yerci, U. Serincan, I. Dogan, S. Tokay, M. Genisel, A. Aydinli, and R. Turan, J. Appl. Phys. 100, 074301 (2006).

${ }^{35}$ G. Scamarcio, M. Lugará, and D. Manno, Phys. Rev. B 45, 13792 (1992).

${ }^{36}$ C. M. Hessel, J. Wei, D. Reid, H. Fujii, M. C. Downer, and B. A. Korgel, J. Phys. Chem. Lett. 3, 1089 (2012).

${ }^{37}$ R. J. Martín-Palma, L. Pascual, P. Herrero, and J. M. Martínez-Duart, Appl. Phys. Lett. 81, 25 (2002).

${ }^{38}$ R. Guerra, E. Degoli, and S. Ossicini, Phys. Rev. B 80, 155332 (2009).

${ }^{39}$ D. E. Yilmaz, C. Bulutay, and T. Çăgin, Appl. Phys. Lett. 94, 191914 (2009); in their calculations, the authors employ the matrix embedment as insertion of a crystalline core into a void in the $\mathrm{SiO}_{2}$ matrix with exactly the same volume as the inserted nanocrystals; therefore, the matrixinduced strain discussed here is not included in these calculations.

${ }^{40}$ I. Yassievich, A. Moskalenko, and A. Prokofiev, Mater. Sci. Eng., C 27, 1386 (2007).

${ }^{41} \mathrm{P}$. Yu and M. Cardonna, Fundamentals of Semiconductors (Springer Berlin, 1996).

${ }^{42}$ R. Cherian, C. Gerard, P. Mahadevan, N. T. Cuong, and R. Maezono, Phys. Rev. B 82, 235321 (2010).

${ }^{43}$ J. Hu and I. Spain, Solid State Commun. 51, 263 (1984).

${ }^{44}$ See supplementary material at http://dx.doi.org/10.1063/1.4756696 for experimental details of the measurements presented in this article, including the used samples.

${ }^{45}$ R. Rölver, O. Winkler, M. Först, B. Spangenberg, and H. Kurz, Microelectron. Reliab. 45, 915 (2005); the authors identify a defect band in the PL of their samples, see [R. Rölver, M. Forst, O. Winkler, B. Spangenberg, and H. Kurz, J. Vac. Sci. Technol. A 24, 141 (2006)]; therefore, the PL spectra in Ref. 45 were fitted with two Gaussians with one fixed at the PL position of the defect and only the SiNC-related peak was plotted in Fig. 5(b).

${ }^{46}$ M. Ray, N. R. Bandyopadhyay, U. Ghanta, R. F. Klie, A. K. Pramanick, S. Das, S. K. Ray, and S. M. Hossain, J. Appl. Phys. 110, 094309 (2011).

${ }^{47}$ The shrinking will apply only for temperatures above the temperature interval of anomalous thermal expansion, which is between $18-120 \mathrm{~K}$ for pure silicon. However, in nanocrystals, this region can be expected to shift to much narrower temperature interval, see Ref. 48, and therefore anomalous thermal expansion can be neglected in the simple-case reasoning.

${ }^{48}$ E. S. Freitas Neto, N. O. Dantas, S. W. da Silva, P. C. Morais, M. A. P. da Silva, A. J. D. Moreno, V. López-Richard, G. E. Marques, and C. TralleroGiner, Nanotechnology 23, 125701 (2012). 